\title{
Impact of RNF213 Founder Polymorphism (p.R4810K) on the Postoperative Development of Indirect Pial Synangiosis After Direct/indirect Combined Revascularization Surgery for Adult Moyamoya Disease.
}

\section{Masaki Ito ( $\sim$ masakiitou-nsu@umin.ac.jp)}

Hokkaido University Graduate School of Medicine School of Medicine: Hokkaido Daigaku Daigakuin Igaku Kenkyuin https://orcid.org/0000-0002-8032-7198

\section{Masahito Kawabori}

Hokkaido University Graduate School of Medicine School of Medicine: Hokkaido Daigaku Daigakuin Igaku Kenkyuin

\section{Taku Sugiyama}

Hokkaido University Graduate School of Medicine School of Medicine: Hokkaido Daigaku Daigakuin Igaku Kenkyuin

\section{Kikutaro Tokairin}

Hokkaido University Graduate School of Medicine School of Medicine: Hokkaido Daigaku Daigakuin Igaku Kenkyuin

\section{Ryota Tatezawa}

Hokkaido University Graduate School of Medicine School of Medicine: Hokkaido Daigaku Daigakuin Igaku Kenkyuin

\section{Haruto Uchino}

Hokkaido University Graduate School of Medicine School of Medicine: Hokkaido Daigaku Daigakuin Igaku Kenkyuin

\section{Ken Kazumata}

Hokkaido University Graduate School of Medicine School of Medicine: Hokkaido Daigaku Daigakuin Igaku Kenkyuin

\section{Kiyohiro Houkin}

Hokkaido University Graduate School of Medicine School of Medicine: Hokkaido Daigaku Daigakuin Igaku Kenkyuin

\section{Miki Fujimura}

Hokkaido University Graduate School of Medicine School of Medicine: Hokkaido Daigaku Daigakuin Igaku Kenkyuin

\section{Research Article}


Keywords: Moyamoya, Magnetic Resonance Imaging, Polymorphism, Revascularization, RNF213, Vascular disorders

Posted Date: August 11th, 2021

DOI: https://doi.org/10.21203/rs.3.rs-781282/v1

License: (c) (1) This work is licensed under a Creative Commons Attribution 4.0 International License. Read Full License

Version of Record: A version of this preprint was published at Neurosurgical Review on February 7th, 2022. See the published version at https://doi.org/10.1007/s10143-022-01749-9. 


\section{Abstract}

Background

Direct superficial temporal artery (STA) to middle cerebral artery (MCA) anastomosis combined with indirect pial synangiosis provides favorable surgical collaterals for Moyamoya disease (MMD), especially in adults; however, factors leading to the development of each direct and indirect collateral are not well documented.

Objective

To investigate the association between RNF213 founder polymorphism ( $\mathrm{p} . \mathrm{R} 4810 \mathrm{~K}$ ) and each direct and indirect collateral development.

Methods

By qualitative and quantitative evaluations of direct and indirect surgical collaterals using time-of-flight MR angiography, postoperative development of each type of bypass was evaluated independently into two categories. Multivariate logistic regression analysis was performed to study the contributing factors for the development of each surgical collateral.

Results

Excellent development of postoperative direct and indirect bypass was observed in 65 hemispheres (70\%) by qualitative evaluation, which was confirmed by quantitative evaluation. Multivariate logistic regression analysis of excellent indirect bypass development revealed a significant positive correlation with the p.R4810K (odds ratio, OR4.0; 95\%-confidence interval, $\mathrm{Cl} 1.2-16)$, advanced MR angiographic stage (OR9.5; 95\% Cl 1.7-73), and preoperative middle meningeal artery caliber (OR6.8; 95\% $\mathrm{Cl} 1.8-35)$, but a significant negative correlation was found with the excellent direct bypass development (OR0.17; 95\% $\mathrm{Cl} 0.03-0.75)$. No significant correlation was observed between excellent direct bypass development and the p.R4810K (OR0.95; 95\% Cl 0.37-2.4).

Conclusion

Excellent development of indirect collaterals after STA-MCA anastomosis combined with indirect pial synangiosis occurs more frequently in adult MMD with the RNF213 founder polymorphism, suggesting a role of the p.R4810K variant for marked in-growth of indirect collaterals and the utility of preoperative genetic analysis.

\section{Introduction}

Moyamoya disease (MMD) is characterized by progressive stenosis of the terminal portion of the internal carotid arteries, accompanied by the formation of abnormally dilated, fragile perforators at the base of the brain. $[6,17$, 28 , 30] Direct superficial temporal artery (STA) to middle cerebral artery (MCA) anastomosis is accepted worldwide as the primary treatment of choice aiming at improving cerebral blood flow and surgical revascularization for symptomatic ischemic and hemorrhagic presentations of MMD. [16, 24] While direct STAMCA anastomosis offers the advantage of immediate revascularization, indirect bypass effectively induces the in-growth of new collaterals to the underlying cerebral cortex overtime. $[17,28]$ In the combined setting using both direct and indirect bypass, the advantages of both bypass procedures are expected, with a perioperative 
stroke risk of $4.7 \%$ per surgery but a favorable long term clinical outcome. $[14,19]$ Several studies demonstrated a reciprocal relationship between the direct and indirect bypass with a wider extent of surgical revascularization in the context of a combined setting. $[1,2,33,34]$ Very recently, the association between a RNF213 founder mutation for east Asian MMD (p.R4810K) [13, 21] and postoperative development of surgical collaterals has been demonstrated,[27] however, it is still obscure for which type of surgical collateral (i.e., direct or indirect or both?) the RNF213 founder mutation is responsible.

The extent of revascularization after Moyamoya bypass surgery is traditionally examined by catheter carotid angiography. [2, 22] In the early 2000s, Yoon et al.[35] and Honda et al.[22] reported the usefulness of magnetic resonance (MR) angiography to evaluate the development of the external carotid artery tributaries, including the STA, middle meningeal artery (MMA), and deep temporal artery (DTA) after direct and/or indirect bypass surgery for MMD. Recent studies using time-of-flight (TOF) MR angiography or its source image provided insights into the discernable and sequential roles in direct and/or indirect bypass in MMD. [1, 34] To comprehensively investigate the clinical and genetic factors associated with the induction of each direct and indirect surgical collaterals, we retrospectively examined our institutional records and MR angiography for adult patients undergoing combined direct/indirect bypass for MMD.

\section{Materials And Methods}

Below are the main methods necessary to comprehend the results. Details were provided in the online-only supplementary information.

\section{Study Population}

This study included all adult MMD patients (>16 years of age at the surgery) who consented to genetic analysis and underwent repeat MR imaging within 3 years after combined direct/indirect bypass $[9,18]$ between 2005 and 2019 at our hospital. quasi-MMD was excluded. The Houkin MR angiographical stage/grading system was used to stratify the angiographical stage of MMD (ranging 1 to 4 [most advanced]). [10] Cerebrovascular reactivity to acetazolamide was evaluated quantitatively as previously reported. [33] In accordance with an institutional review board-approved protocol (number14-053), medical records were retrospectively reviewed to gather demographic information, age at the surgery, symptoms at presentation, comorbid conditions before surgery, and results of radiographic studies (Please see supplementary table 1).

Evaluation of the Postoperative Development of Direct and Indirect Surgical Collaterals by MR angiography

Evaluation of the postoperative development of direct and indirect surgical collaterals was performed qualitatively and quantitatively using TOF MR angiography and its source images, respectively, by a previously reported protocol with minor modifications $[33,34]$. In brief, MR angiography at two time points acquired before and 6 to 36 months after surgery were reviewed and compared by neurosurgeons (M. I. and T.S.) blinded to the results of genetic testing. For qualitative evaluation, [33] postoperative development of each direct and indirect surgical collaterals were dichotomized into excellent or not, respectively. Thus, the development of direct surgical collaterals was evaluated by the development of the STA, while that of indirect surgical collaterals was evaluated by the development of the MMA and DTA. For quantitative evaluation, we reviewed MR angiography source images and measured the calibers of the STA, MMA, and DTA as previously reported.[34] The caliber change ratios (CCRs) of post to preoperative calibers were calculated for each artery. 
Written-informed consent was received for genetic analysis from MMD patients or their guardians. In accordance with the institutional review board-approved protocol, genetic analysis was conducted at the Department of Neurosurgery of Hokkaido University by K.T. and R.T. who were blinded to clinical data. Taqman single nucleotide polymorphism genotyping assay was employed to determine the allele type for RNF213 founder mutation (p.R4810K).

Data Analysis

Continuous or rank variables were described as the mean or median with standard deviation or range. Dichotomous or categorical variables were expressed as the ratio or frequency. Continuous or rank variables were compared between two groups by the unpaired-t test or Mann-Whitney test. Dichotomous or categorical variables were compared by Fisher's exact test. For multiple comparisons, two-way repeated measures or ordinary analysis of variance followed by a post-hoc test was used, as appropriate. To assess the correlation between the excellent development of each surgical collateral and multiple clinical and genetic variables, multivariate logistic regression analysis was performed using the stepwise forward parameter selection that achieved significance levels of $P<0.1$ in the univariate analysis. All clinical and genetic factors for the multivariate analysis are listed in the main Tables. The level of significance was set at $P<0.05$. GraphPad Prism (version 9.1.1, San Diego, CA, USA) was used for these analyses.

\section{Results}

\section{Study Population}

During the study period, 110 adult patients with 160 hemispheres underwent STA-MCA anastomosis combined with indirect pial synangiosis using vascularized tissue, including dura mater and temporal muscle. Of these, 48 were excluded from analysis due to lack of genetic testing, missing follow-up MR imaging within 6 to 36 months after surgery, or the diagnosis of quasi-MMD ( $n=43,2$, and 3 patients, respectively). Consequently, 62 patients (56\%) with 93 operated hemispheres (58\%) were included in the analysis: 47 female and 15 male patients with an average age of 42 years. RNF213 founder mutation (p.R4810K) was detected in 40 patients (65\%) with 59 hemispheres (63\%) in this series (Figure 1A). There was no significant difference in baseline characteristics between RNF213-mutant (MT) and -wild type (WT) groups, except in familial occurrence and a comorbid condition of hypertension (Supplementary Table. 2). A representative patient who was a heterozygote for the RNF213 founder mutation is shown in Figure 1B-E.

Qualitative and Quantitative MR Angiography Evaluations

Of all 93 hemispheres enrolled in this study, excellent development of postoperative direct and indirect surgical collaterals was observed in $65(70 \%)$ after the combined bypass with a mean follow-up period of $319 \pm 140$ days by qualitative evaluation. In terms of the relationship of the postoperative development between direct and indirect bypass, dual/equal development was most frequently observed (62\% of the operated hemisphere). Thus, postoperative direct- (STA) or indirect- (MMA and/or DTA) dominant development was observed in 44 and 14 hemispheres, respectively, when evaluated in the above-mentioned follow-up period. By quantitative MR angiography evaluation of all 93 hemispheres, a significant increase was observed in the caliber of the STA after 
surgery (pre: $1.8 \pm 0.35 \mathrm{~mm}$; post: $2.5 \pm 0.65 \mathrm{~mm}, \mathrm{P}<0.0001)$. The caliber of the MMA and DTA also significantly increased (pre: $1.6 \pm 0.44 \mathrm{~mm}$; post: $1.8 \pm 0.50 \mathrm{~mm}, \mathrm{P}<0.0001$ in MMA; pre: $0.98 \pm 0.33 \mathrm{~mm}$; post: $1.6 \pm 0.60 \mathrm{~mm}$, $\mathrm{P}<0.0001$, respectively). The CCR of post to preoperative-calibers for the STA was significantly higher $(1.5 \pm 0.42$ vs $1.0 \pm 0.28, P<0.0001)$ in the excellent direct bypass development group than in the non-excellent group. The CCRs for the DTA $(2.0 \pm 0.89$ vs $1.2 \pm 0.53, P<0.0001)$ and MMA $(1.3 \pm 0.31$ vs $1.0 \pm 0.29, P=0.042)$ were also significantly higher in the excellent indirect bypass development group.

We further analyzed the association between direct or indirect bypass development and RNF213 founder polymorphism (p.R4810K). Of note, excellent indirect bypass development was observed more frequently in the RNF213-MT group (78\%, 46/59 hemispheres) than in the RNF213-WT group (56\%, 19/34 hemispheres), with a significant difference $(P=0.035)$ (Figure $2 A)$. Excellent direct bypass development was observed in 40/59 hemispheres (68\%) and 24/34 hemispheres (71\%) of the RNF213-MT and -WT groups, respectively, with no significant difference $(P=0.82)$. Multiple comparisons of the CCRs demonstrated a significant difference in the DTA, but not in the STA or MMA, between the two groups (Figure $2 \mathrm{~B}$ ). Thus, the CCR for the DTA was significantly higher in the RNF213-MT group (1.9 \pm 1.0$)$ than in the RNF213-WT group $(1.4 \pm 0.52, \mathrm{P}=0.0007)$.

Factors Correlated with Excellent Development of Indirect and Direct Collaterals After Combined Bypass

To identify clinical and genetic factors that may underlie excellent revascularization after combined bypass in adult MMD, we examined which factors correlated with the excellent development of indirect and direct collaterals (Table 1 and 2). Multivariate logistic regression analysis for excellent indirect bypass development revealed a significant positive correlation with RNF213 founder mutation (adjusted odds ratio (OR), 4.0), advanced MR angiographic stage (adjusted OR, 13 in stage 3; 9.5 in stage 4), and preoperative caliber of the MMA (adjusted OR, 6.8), whereas a significant negative correlation was noted with excellent direct bypass development (adjusted OR, 0.17). On the other hand, no significant correlation was observed between excellent direct bypass development and RNF213 founder mutation. Multivariate logistic regression analysis for excellent direct bypass development revealed a significant negative correlation with excellent indirect bypass development (adjusted OR, 0.23) and the comorbid condition of dyslipidemia (adjusted OR, 0.27). Please see supplementary figure 1 and 2 supporting these results.

\section{Discussion}

To our knowledge, this is the first study to demonstrate that the RNF213 gene polymorphism (p.R4810K) plays a role in the postoperative development of indirect, but not direct surgical collaterals after direct STA-MCA anastomosis combined with indirect pial synangiosis for adult patients with MMD. We were able to document this relationship by qualitative and quantitative evaluation of repeated TOF MR angiography during the short to mid-term follow-up period (6 to 36 months after surgery). Although evaluation of calibers of donor and recipient arteries by MR angiography rather than catheter angiography is suboptimal, the usefulness of TOF MR angiography for the evaluation of the external carotid artery tributaries after Moyamoya bypass surgery has been validated by several researchers either in a direct,[8] indirect [35] or combined bypass setting.[34] Our observation is consistent with previously published studies that favorable development of both bypass types over time is most frequent after combined direct and indirect bypass in adult MMD.[1, 33, 34] 
To further determine key factors responsible for indirect and direct bypass development, respectively, we focused on significant clinical and genetic factors correlating with the excellent postoperative development of surgical collaterals by multivariate logistic regression analyses. We identified a panel of excellent revascularizationrelated factors for each indirect and direct bypass development after combined bypass for adult MMD (main Tables). Of note, 1) p.R4810K variant, 2) preoperative advanced MR angiographical stage, and 3) preoperative larger caliber of the MMA were positively, and 4) postoperative excellent direct bypass development was negatively correlated with excellent indirect bypass development. This is partly current knowledge. Thus, advanced angiographical stage, preoperative trans-dural collateral vessels (i.e., MMA), and heterozygous p.R4810K variant are known radiographic and genetic biomarkers of the increased capacity of postoperative surgical collateral production. [27, 29] In addition to the current knowledge, our study suggests significant correlation between p.R4810K and excellent indirect, but not direct bypass development after combined bypass, although its underlying mechanism is unclear based on our study, which is briefly discussed below. Thus, it remains unclear how p.R4810K plays a pathological role in MMD (i.e., why earlier disease onset, higher disease severity, [15, 25] and prolonged/delayed cerebral hyperperfusion after STA-MCA anastomosis [31] occur more frequently in MMD with the RNF213 founder mutation?). Earlier experimental studies reported that cellular gene expression analysis of $R N F 213$ in adult human tissues revealed markedly high expression in immune tissues such as the spleen and leukocytes.[13, 23] Genome-wide plasma/serum microRNA profiling $[5,12,32]$ revealed a panel of significant MMD-related plasma/serum microRNAs whose target genes were involved in inflammatory or angiogenesis-related molecular pathways. Bidirectional major pathways that are influential in the inflammatory response potentially causing collateral formation in MMD are 1) the anti-inflammatory cytokine pathway and 2) proinflammatory cytokine pathway activating RNF213. Fujimura et al. (2018) recently showed increased serum production of soluble CD163 and CXCL5 in MMD patients, suggesting the involvement of intrinsic M2 macrophage-related immune reactions.[7] The immune responses associated with angiogenesis are promoted by $\mathrm{M} 2$ macrophages and angiogenic mediators are activated through these anti-inflammatory cytokines.[23] Ohkubo et al. (2015) reported that pro-inflammatory cytokines activated transcription of $R N F 213$ both in vitro and in vivo. p.R4810K variant was more likely linked to the functional deficiency of the RNF213 gene based on markedly high matrix metalloproteinase production upon experimental silencing of RNF213.[26] Bang et al. (2016) reported a marked increase in the blood caveolin-1 level in RNF213 founder mutation carriers.[3] As caveolin-1 negatively regulates proliferation of endothelial cells, but positively regulates endothelial angiogenic function such as tube formation,[20] the increased caveolin-1 levels may accelerate angiogenesis in MMD patients. In vivo experimental study demonstrated increased angiogenesis in mice lacking RNF213 after chronic hind-limb ischemia, [11] suggesting a role of RNF213 abnormality in the development of pathological vascular networks in chronic ischemia. Taken together, our observation of marked angiogenesis represented by excellent indirect bypass development in adult MMD patients with P.R4810K variant can be explained bidirectionally by the loss-of-function (i.e., marked angiogenesis by lacking RNF213 gene function) and gain-of-function (i.e., marked angiogenesis through RNF213 mutation) mechanisms of the RNF213 gene, which is the next question to be addressed.

Another novel finding demonstrated in this study is that the comorbid condition of dyslipidemia was negatively correlated with excellent direct bypass development. Recently, Church and Steinberg et al. (2020) reported an association between hyperlipidemia and radiological progression of unilateral type to bilateral type in MMD, with possible explanations including synergistic effects of increased lipids in the underlying moyamoya vasculopathy and the inadvertent inclusion of cerebral atherosclerotic disease in the study population.[4] One possible

Page $7 / 18$ 
explanation for our finding may also be the inadvertent involvement of atherosclerosis in our MMD patients, which leads to a poorer condition of both the donor and recipient arterial wall, resulting in poor long-term patency.

Limitation

Our study is limited by the following several points. First, our study did not include pediatric subgroup of MMD. As we previously reported that almost all (95\%) pediatric MMD exhibited effective indirect revascularization after combined bypass,[33] we only investigated adult MMD in this study. Second, catheter angiography follow-up was not available in the most bypass surgeries in this study, although measurement of vessel calibers by catheter angiography is optimal. Last, consistent hemodynamic evaluations were not available for all patients using single-photon emission computed tomography or positron emission tomography. We do not consider these limitations to affect the interpretation of the results, but further studies are warranted.

\section{Conclusions}

We found that the excellent development of postoperative indirect pial synangiosis after combined direct and indirect bypass occurs more frequently in adult MMD patients with the RNF213 founder mutation (p.R4810K) allele. This confirms a novel clinical role of the RNF213 founder polymorphism in the marked angiogenesis via indirect pial synangiosis in adult patients with $M M D$, and suggests the utility of preoperative genetic analysis for RNF213 polymorphism in MMD.

\section{Declarations}

Funding: This study was partially supported by Japanese Society for the Promotion of Science KAKENHI grant number $19 \mathrm{H} 03765$ (M.I.) and 20K09362 (M.F.). All authors declare that there is no personal or institutional financial interest in drugs, materials, or devices described in this article.

Conflicts of interest/Competing interests: On behalf of all authors, the corresponding author state that the authors report no conflict of interest concerning the materials or methods used in this study or the findings specified in this paper.

Availability of data and material: Data supporting the findings of this study are available from the corresponding author on reasonable request.

Code availability: Not applicable

Authors' contributions: Conception and design: Fujimura M, Ito M, and Kawabori M. Acquisition of data: Ito M., Sugiyama T, Tokairin K, Tatezawa R. Analysis and interpretation of data: Ito M, Kawabori M, Sugiyama T, Tokairin $\mathrm{K}$, Tatezawa R, Uchino H, and Fujimura M. Drafting the article: Ito M. Critically revising the article: Kazumata K, and Fujimura M. Statistical analysis: Ito M. Study supervision: Kazumata K and Houkin K.

Ethics approval: The present study was approved by the Hokkaido University Graduate School of Medicine medical ethics committee on human experimentation, including genetic analysis (14-053). 
Consent to participate: Written informed consent was obtained from all participants (or guardians) to participate in this study.

Consent to publication: Written informed consent was obtained from all participants (or guardians) whose individual data were presented for publication in this study in any form.

\section{Acknowledgements}

We would like to acknowledge Ms. Rika Nagashima and Ms. Ikuyo Hata for their administrative assistance. We also thank Dr. Hiroyuki Kohno, Dr Kanako Hatanaka, and Ms. Miyuki Kasegai for their technical support in the genetic analysis.

\section{References}

1. Amin-Hanjani S, Singh A, Rifai H, Thulborn KR, Alaraj A, Aletich V, Charbel FT (2013) Combined direct and indirect bypass for moyamoya: Quantitative assessment of direct bypass flow over time. Neurosurgery 73:962968

2. Bang JS, Kwon OK, Kim JE, Kang HS, Park H, Cho SY, Oh CW (2012) Quantitative angiographic comparison with the OSIRIS program between the direct and indirect revascularization modalities in adult moyamoya disease. Neurosurgery 70:625-633

3. Bang OY, Chung JW, Kim SJ, Oh MJ, Kim SY, Cho YH, Cha J, Yeon JY, Kim KH, Kim GM, Chung CS, Lee KH, Ki CS, Jeon P, Kim JS, Hong SC, Moon GJ (2016) Caveolin-1, Ring finger protein 213, and endothelial function in Moyamoya disease. Int J Stroke 11:999-1008

4. Church EW, Bell-Stephens TE, Bigder MG, Gummidipundi S, Han SS, Steinberg GK (2020) Clinical course of unilateral moyamoya disease. Neurosurgery 87:1262-1268

5. Dai D, Lu Q, Huang Q, Yang P, Hong B, Xu Y, Zhao W, Liu J, Li Q (2014) Serum miRNA Signature in Moyamoya Disease. PLoS One 9:e102382

6. Fujimura M, Bang OY, Kim JS (2016) Moyamoya Disease. Front Neurol Neurosci 40:204-220

7. Fujimura M, Fujimura T, Kakizaki A, Sato-Maeda M, Niizuma K, Tomata Y, Aiba S, Tominaga T (2018) Increased serum production of soluble CD163 and CXCL5 in patients with moyamoya disease: Involvement of intrinsic immune reaction in its pathogenesis. Brain Res 1679:39-44

8. Honda M, Kitagawa N, Tsutsumi K, Morikawa M, Nagata I, Kaminogo M (2005) Magnetic resonance angiography evaluation of external carotid artery tributaries in moyamoya disease. Surg Neurol 64:325-330

9. Houkin K, Kamiyama H, Takahashi A, Kuroda S, Abe H (1997) Combined revascularization surgery for childhood moyamoya disease: STA-MCA and encephalo-duro-arterio-myo-synangiosis. Child's Nerv Syst 13:2429

10. Houkin K, Nakayama N, Kuroda S, Nonaka T, Shonai T, Yoshimoto T (2005) Novel magnetic resonance angiography stage grading for moyamoya disease. Cerebrovasc Dis 20:347-54 
11. Ito A, Fujimura M, Niizuma K, Kanoke A, Sakata H, Morita-Fujimura Y, Kikuchi A, Kure S, Tominaga T (2015) Enhanced post-ischemic angiogenesis in mice lacking RNF213; a susceptibility gene for moyamoya disease. Brain Res 1594:310-20

12. Ito M, Uchino $\mathrm{H}$ (2020) Detection and quantification of microRNAs (miRNAs) and high-throughput miRNA profiling. In: Epigenetics Methods. Elsevier, pp 479-493

13. Kamada F, Aoki Y, Narisawa A, Abe Y, Komatsuzaki S, Kikuchi A, Kanno J, Niihori T, Ono M, Ishii N, Owada Y, Fujimura M, Mashimo Y, Suzuki Y, Hata A, Tsuchiya S, Tominaga T, Matsubara Y, Kure S (2011) A genome-wide association study identifies RNF213 as the first Moyamoya disease gene. J Hum Genet 56:34-40

14. Kazumata K, Ito M, Tokairin K, Ito Y, Houkin K, Nakayama N, Kuroda S, Ishikawa T, Kamiyama H (2014) The frequency of postoperative stroke in moyamoya disease following combined revascularization: a singleuniversity series and systematic review. J Neurosurg 121:432-440

15. Kim EH, Yum MS, Ra YS, Park JB, Ahn JS, Kim GH, Goo HW, Ko TS, Yoo HW (2016) Importance of RNF213 polymorphism on clinical features and long-term outcome in moyamoya disease. J Neurosurg 124:1221-1227

16. Kuroda S (2021) Overview of Surgical Revascularization and Long-Term Outcome in Japan. In: Moyamoya Disease: Current Knowledge and Future Perspectives. Springer Singapore, pp 215-227

17. Kuroda S, Houkin K (2008) Moyamoya disease: current concepts and future perspectives. Lancet Neurol 7:1056-1066

18. Kuroda S, Houkin K, Ishikawa T, Nakayama N, Iwasaki Y (2010) Novel bypass surgery for moyamoya disease using pericranial flap: Its impacts on cerebral hemodynamics and long-term outcome. Neurosurgery 66:1093-1101

19. Kuroda S, Nakayama N, Yamamoto S, Kashiwazaki D, Uchino H, Saito H, Hori E, Akioka N, Kuwayama N, Houkin K (2021) Late (5-20 years) outcomes after STA-MCA anastomosis and encephalo-duro-myo-arteriopericranial synangiosis in patients with moyamoya disease. J Neurosurg 134:909-916

20. Liu J, Wang XB, Park DS, Lisanti MP (2002) Caveolin-1 expression enhances endothelial capillary tubule formation. J Biol Chem 277:10661-10668

21. Liu W, Morito D, Takashima S, Mineharu Y, Kobayashi H, Hitomi T, Hashikata H, Matsuura N, Yamazaki S, Toyoda A, Kikuta K, Takagi Y, Harada KH, Fujiyama A, Herzig R, Krischek B, Zou L, Kim JE, Kitakaze M, Miyamoto S, Nagata K, Hashimoto N, Koizumi A (2011) Identification of RNF213 as a Susceptibility Gene for Moyamoya Disease and Its Possible Role in Vascular Development. PLoS One 6:e22542

22. Matsushima T, Inoue T, Suzuki SO, Fujii K, Fukui M, Hasuo K (1992) Surgical treatment of moyamoya disease in pediatric patients-comparison between the results of indirect and direct revascularization procedures. Neurosurgery 31:401-405

23. Mikami T, Suzuki H, Komatsu K, Mikuni N (2019) Influence of inflammatory disease on the pathophysiology of moyamoya disease and quasi-moyamoya disease. Neurol Med Chir (Tokyo) 59:361-370 
24. Miyamoto S, Yoshimoto T, Hashimoto N, Okada Y, Tsuji I, Tominaga T, Nakagawara J, Takahashi JC (2014) Effects of extracranial-intracranial bypass for patients with hemorrhagic moyamoya disease: results of the Japan Adult Moyamoya Trial. Stroke 45:1415-21

25. Miyatake S, Miyake N, Touho H, Nishimura-Tadaki A, Kondo Y, Okada I, Tsurusaki Y, Doi H, Sakai H, Saitsu H, Shimojima K, Yamamoto T, Higurashi M, Kawahara N, Kawauchi H, Nagasaka K, Okamoto N, Mori T, Koyano S, Kuroiwa Y, Taguri M, Morita S, Matsubara Y, Kure S, Matsumoto N (2012) Homozygous c.14576G>A variant of RNF213 predicts early-onset and severe form of moyamoya disease. Neurology 78:803-810

26. Ohkubo K, Sakai Y, Inoue H, Akamine S, Ishizaki Y, Matsushita Y, Sanefuji M, Torisu H, Ihara K, Sardiello M, Hara T (2015) Moyamoya disease susceptibility gene RNF213 links inflammatory and angiogenic signals in endothelial cells. Sci Rep 5:13191

27. P G, X Y, X L, X D, J W, R W, Y Z, D Z, Q Z, J Z (2019) Association between p.R4810K Variant and Postoperative Collateral Formation in Patients with Moyamoya Disease. Cerebrovasc Dis 48:77-84. doi: $10.1159 / 000503250$

28. Scott RM, Smith ER (2009) Moyamoya Disease and Moyamoya Syndrome. N Engl J Med 360:1226-1237

29. Storey A, Scott RM, Robertson R, Smith E (2017) Preoperative transdural collateral vessels in moyamoya as radiographic biomarkers of disease. J Neurosurg Pediatr 19:289-295

30. Suzuki J, Takaku A (1969) Cerebrovascular "moyamoya" disease. Disease showing abnormal net-like vessels in base of brain. Arch Neurol 20:288-299

31. Tashiro R, Fujimura M, Katsuki M, Nishizawa T, Tomata Y, Niizuma K, Tominaga T (2020) Prolonged/delayed cerebral hyperperfusion in adult patients with moyamoya disease with RNF213 gene polymorphism c.14576G>A (rs112735431) after superficial temporal artery-middle cerebral artery anastomosis. J Neurosurg 1-8

32. Uchino H, Ito M, Kazumata K, Hama Y, Hamauchi S, Terasaka S, Sasaki H, Houkin K (2018) Circulating miRNome profiling in Moyamoya disease-discordant monozygotic twins and endothelial microRNA expression analysis using iPS cell line. BMC Med Genomics 11:72

33. Uchino H, Kim JH, Fujima N, Kazumata K, Ito M, Nakayama N, Kuroda S, Houkin K (2017) Synergistic interactions between direct and indirect bypasses in combined procedures: The significance of indirect bypasses in moyamoya disease. Neurosurgery 80:201-209

34. Uchino H, Yamamoto S, Kashiwazaki D, Akioka N, Kuwayama N, Noguchi K, Kuroda S (2021) Using postoperative remodeling of donor arteries on MR angiography to predict the development of surgical collaterals in moyamoya disease. J Neurosurg 134:1-9

35. Yoon HK, Shin HJ, Lee M, Byun HS, Na DG, Han BK (2000) MR angiography of moyamoya disease before and after encephaloduroarteriosynangiosis. Am J Roentgenol 174:195-200

\section{Tables}


TABLE 1 Correlation of Postoperative Indirect bypass development with Clinical and Genetic Variables 


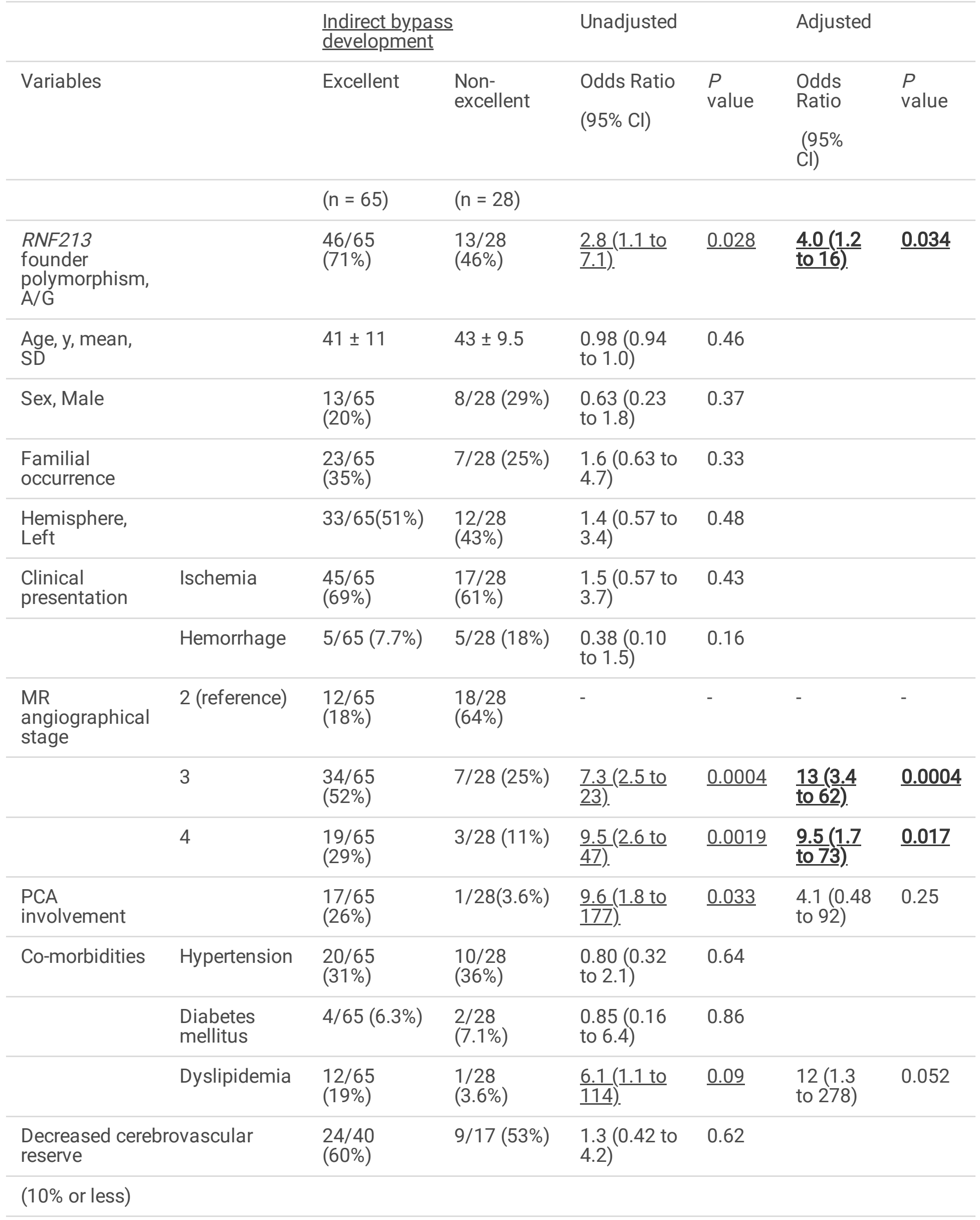




$\begin{array}{lllllll}\text { Direct bypass development, } & 40 / 65 & 25 / 28 & \underline{0.19}(\underline{0.043} & \underline{0.013} & \underline{\mathbf{0 . 1 7}} \\ \text { Excellent } & (62 \%) & (89 \%) & \underline{\text { to } 0.62)} & \underline{\mathbf{0 . 0 3}} \text { to } & \underline{\mathbf{0 . 0 2 9}} \\ & & & \underline{0.75})\end{array}$

Preoperative donor artery diameter

\begin{tabular}{|c|c|c|c|c|c|c|c|}
\hline \multirow[t]{4}{*}{ mm, mean, SD } & STA & $1.8 \pm 0.36$ & $1.9 \pm 0.32$ & $\begin{array}{l}0.83(0.23 \\
\text { to } 3.0)\end{array}$ & 0.78 & & \\
\hline & MMA & $1.6 \pm 0.45$ & $1.4 \pm 0.40$ & $\left.\frac{3.9}{14}\right)$ (1.3 to & $\underline{0.023}$ & $\frac{6.8}{\text { to } 35)}$ & $\underline{0.010}$ \\
\hline & DTA & $1.0 \pm 0.36$ & $0.95 \pm 0.34$ & $\begin{array}{l}1.6(0.41 \text { to } \\
6.7)\end{array}$ & 0.50 & & \\
\hline & BA & $3.0 \pm 0.47$ & $3.1 \pm 0.61$ & $\begin{array}{l}0.67(0.27 \\
\text { to } 1.6)\end{array}$ & 0.36 & & \\
\hline \multicolumn{2}{|c|}{$\begin{array}{l}\text { Days after bypass for the } \\
\text { assessment }\end{array}$} & $305 \pm 127$ & $350 \pm 167$ & $\begin{array}{l}1.0(1.0 \text { to } \\
1.0)\end{array}$ & 0.17 & & \\
\hline
\end{tabular}

Abbreviations: RNF213, ring finger protein 213; Cl, confidence interval; SD, standard deviation; PCA, posterior cerebral artery; STA, superficial temporal artery; MMA, middle meningeal artery; DTA, deep temporal artery; BA, basilar artery

TABLE 2 Correlation of Postoperative Direct bypass development with Clinical and Genetic Variables 
Direct bypass

Variables development

Unadjusted

$\begin{array}{ll}\text { Excellent } & \text { Non- } \\ & \text { excellent }\end{array}$
Odds Ratio $(95 \% \mathrm{Cl})$

Adjusted

$(\mathrm{n}=65) \quad(\mathrm{n}=28)$

RNF213

founder

polymorphism,

A/G

Age, y, mean,

SD

Sex, Male

Familial

occurrence

Hemisphere,

Left

Clinical

presentation

$\begin{array}{llll}41 / 65 & 18 / 28 & 0.95(0.37 & 0.91 \\ (63 \%) & (64 \%) & \text { to } 2.4) & \end{array}$

$42 \pm 9.9 \quad 42 \pm 11$

$1.0(0.96$ to 0.93

1.0)

$15 / 65$

(23\%)

$6 / 28$

(21\%)

1.1 (0.39 to 0.86

3.4)

$21 / 65$

(32\%)

$9 / 28$

(32\%)

$1.0(0.40$ to 0.99

2.7)

$33 / 65$

(51\%)

$12 / 28$

(43\%)

$1.4(0.57$ to

0.48

3.4)

$42 / 65$

(65\%)

20/28

(71\%)

$0.71(0.26$

0.52

to 1.8$)$

Hemorrhage

$7 / 65$

(11\%)

$3 / 28$

(11\%)

$1.0(0.26$ to

0.99

$5.0)$

MR

angiographical

stage

2 (reference)

23/65

(35\%)

$7 / 28$

(25\%)

3

PCA

involvement

$28 / 65$

(43\%)

$13 / 28$

(46\%)

$0.66(0.22$

0.44

$14 / 65$

(22\%)

$8 / 28$

(29\%)

to 1.9 )

$11 / 65$

(17\%)

$7 / 28(25 \%)$

$0.53(0.15$

to 1.8$)$

Co-morbidities

Hypertension

$21 / 65$

(32\%)

$9 / 28$

(32\%)

$0.61(0.21$

to 1.9$)$

$\begin{array}{lllll}\begin{array}{l}\text { Diabetes } \\ \text { mellitus }\end{array} & \begin{array}{l}3 / 65 \\ (4.6 \%)\end{array} & \begin{array}{l}3 / 28 \\ (11 \%)\end{array} & \begin{array}{l}0.40(0.07 \\ \text { to 2.3) }\end{array} & 0.29 \\ \text { Dyslipidemia } & \begin{array}{l}5 / 65 \\ (7.7 \%)\end{array} & \begin{array}{l}8 / 28 \\ (29 \%)\end{array} & \underline{\underline{0.21}(0.057} & \underline{0.012} \\ & & \underline{0.0 .70}) & \end{array}$

$1.0(0.40$ to 0.99

2.7)

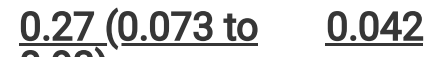

$\underline{0.93}$.

Odds Ratio

$P$

value 
diameter

\begin{tabular}{|c|c|c|c|c|c|}
\hline \multirow[t]{4}{*}{$\mathrm{mm}$, mean, SD } & STA & $\begin{array}{l}1.8 \pm \\
0.32\end{array}$ & $1.9 \pm 0.36$ & $\begin{array}{l}1.2(0.35 \text { to } \\
4.6)\end{array}$ & 0.75 \\
\hline & MMA & $\begin{array}{l}1.5 \pm \\
0.32\end{array}$ & $1.6 \pm 0.49$ & $\begin{array}{l}1.9(0.67 \text { to } \\
5.7)\end{array}$ & 0.24 \\
\hline & DTA & $\begin{array}{l}0.98 \pm \\
0.30\end{array}$ & $\begin{array}{l}0.98 \pm \\
0.34\end{array}$ & $\begin{array}{l}1.1(0.27 \text { to } \\
4.2)\end{array}$ & 0.94 \\
\hline & BA & $\begin{array}{l}3.1 \pm \\
0.51\end{array}$ & $3.0 \pm 0.52$ & $\begin{array}{l}0.79(0.33 \\
\text { to } 1.9)\end{array}$ & 0.60 \\
\hline \multicolumn{2}{|c|}{$\begin{array}{l}\text { Days after bypass for the } \\
\text { assessment }\end{array}$} & $\begin{array}{l}307 \pm \\
105\end{array}$ & $323 \pm 154$ & $\begin{array}{l}1.0(1.0 \\
\text { to } 1.0)\end{array}$ & 0.61 \\
\hline
\end{tabular}

Abbreviations: RNF213, ring finger protein 213; Cl, confidence interval; SD, standard deviation; PCA, posterior cerebral artery;

STA, superficial temporal artery; MMA, middle meningeal artery; DTA, deep temporal artery; BA, basilar artery

\section{Figures}
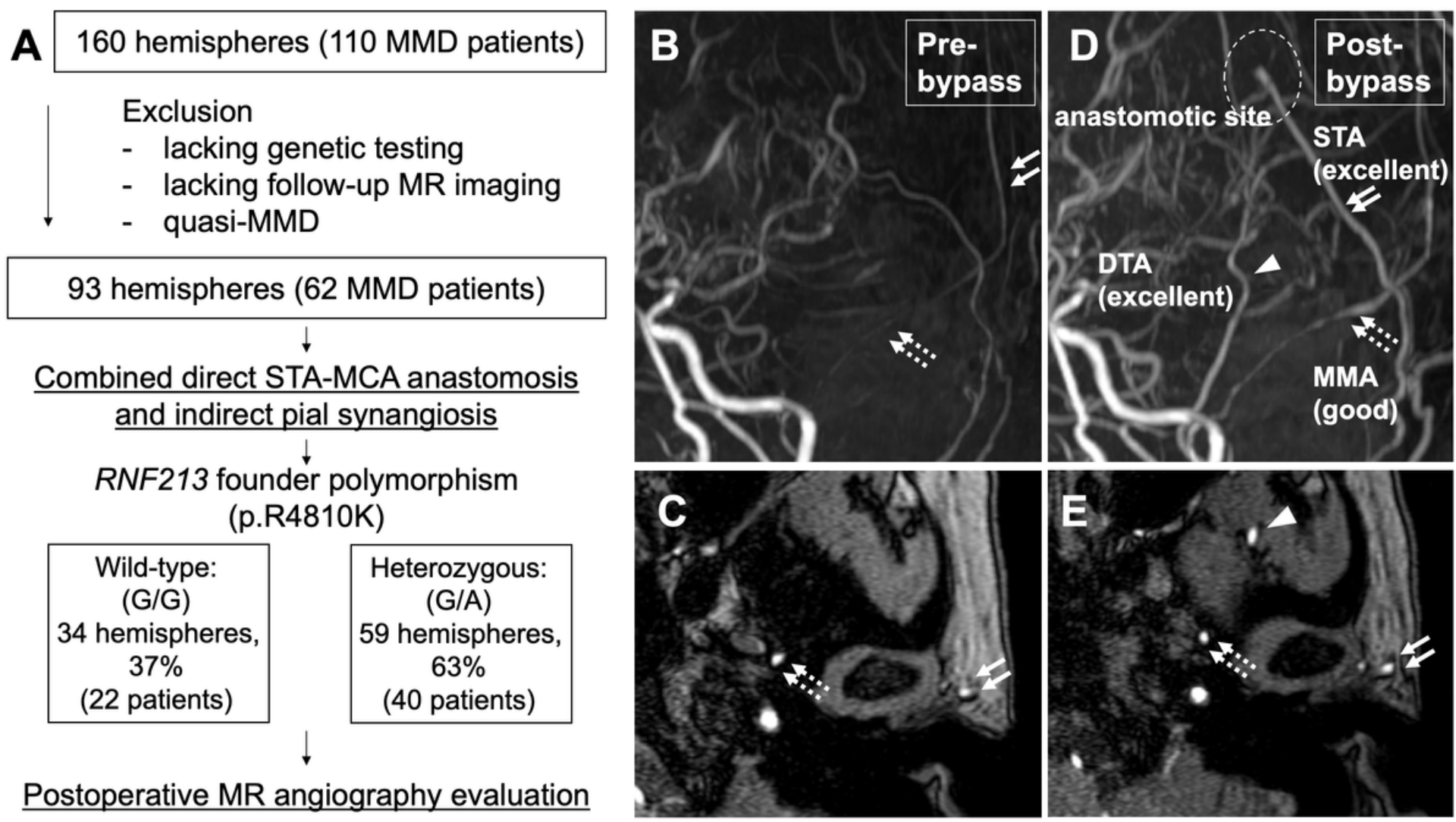

Figure 1

Flow chart for inclusion of the study subjects and representative MR angiography of a study subject before and after combined direct/indirect bypass (A) Flow chart shows the breakdown of inclusion and exclusion of the study subjects. (B-E) MR angiography of a 49-year-old female with Moyamoya disease obtained before and 6 
months after left combined direct and indirect bypass. Preoperative axial time-of-flight MR angiography with maximal intensity projection (MIP) reconstruction (B) and its source image (C) of a 49-year-old MMD female with the RNF213 founder polymorphism (A/G genotype) showed the left superficial temporal artery (STA, double arrows) and middle meningeal artery (MMA, dotted arrows). The deep temporal artery was hardly seen in MIP or its source images preoperatively. (D, E) Follow-up MR angiography obtained 6 months after left STA-MCA anastomosis combined with encephalo-duro-myo-arterio-pericranio-synangiosis demonstrated excellent development of postoperative direct (STA) and indirect (MMA and DTA) surgical collaterals.

A

\section{Qualitative Analysis}

Indirect bypass development

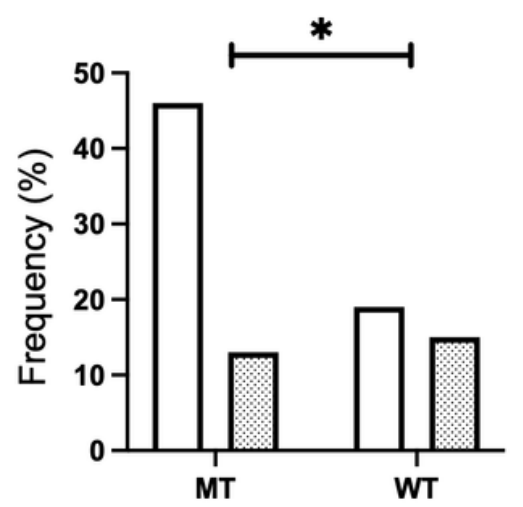

RNF213 founder polymorphism p.R4810K

$\square$ Excellent
Direct bypass development

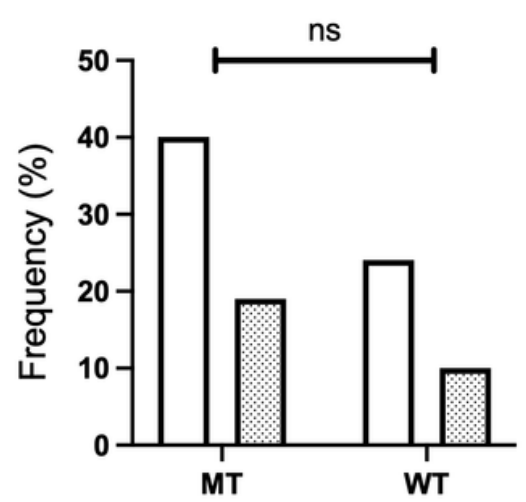

RNF213 founder polymorphism p.R4810K

Non-excellent

\section{Quantitative Analysis}

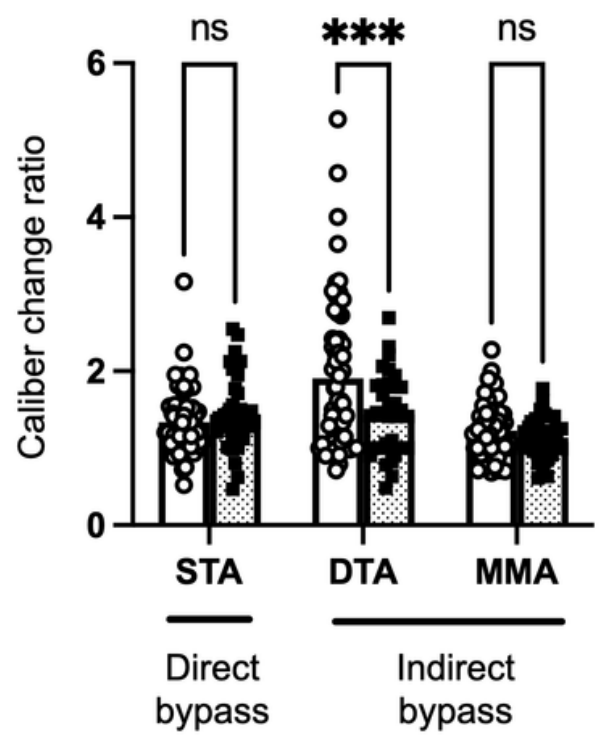

- RNF213 MT - RNF213 WT

Figure 2

Qualitative and quantitative analysis of postoperative direct and indirect collateral development using time-offlight magnetic resonance angiography (A) Interleaved bar graph showing the results of qualitative analysis of the axial time-of-flight MR angiography with maximal intensity projection (MIP) reconstruction before and after combined bypass to evaluate the development of postoperative indirect and direct collaterals in adult MMD. ns, not significant; * $\mathrm{P}<0.05$, Fisher's exact test. (B) Interleaved scatterplot with bars showing the caliber change ratios for the superficial temporary artery (STA), deep temporal artery (DTA), and middle meningeal artery (MMA) from quantitative analysis of the source images of MR angiography before and after combined bypass in adult MMD. ns, not significant; ***P <0.001, two-way ANOVA followed by Bonferroni multiple comparison.

\section{Supplementary Files}

This is a list of supplementary files associated with this preprint. Click to download.

- SuppleTABLE1.pdf 
- SuppleTABLE2.pdf

- Supplementaryfile.docx

- supplfigure1.pdf

- supplfigure2.pdf 